\title{
HIV knowledge, sexual health and sexual behaviour among Black and minority ethnic men who have sex with men in the UK: a cross-sectional study
}

\author{
Rusi Jaspal \\ Trinity House, De Montfort University, Leicester LE2 7BY, UK. \\ Faculty of Arts, Psychology \& Theology, Åbo Akademi University, 20500 Turku, Finland. \\ Barbara Lopes \\ Educação da Universidade de Coimbra, Rua do Colégio Novo, P-301-802 Coimbra, Portugal. \\ Zahra Jamal, Carmen Yap, Ivana Paccoud and Parminder Sekhon \\ NAZ Project London, 30 Black’s Road, London W6 9DT, UK.
}

\begin{abstract}
Background: Black and minority ethnic (BME) men who have sex with men (MSM) face a major burden in relation to HIV infection. Using a cross-sectional correlational survey design, the present study explored the relationships between HIV knowledge and reported sexual health and sexual behaviour in this population. Methods: A convenience sample of 538 BME MSM was recruited in London, Leicester and Leeds: 346 (64\%) self-identified as South Asian, $88(16 \%)$ self-identified as Latin American, 76 (14\%) self-identified as Black, $13(2 \%)$ self-identified as mixed, and 15 (3\%) self-identified as other. Results: HIV knowledge was low across the board, and South Asian MSM manifested the lowest scores. Respondents who perceived their HIV risk to be low possessed the least HIV knowledge. There were interethnic differences in the frequency of gay sauna visits, sex seeking on mobile applications, drug use and attendance at sex parties. Respondents reported a high frequency of racism and discrimination, with Black MSM reporting highest frequency. Conclusions: There is an urgent need to raise awareness of HIV in BME MSM, and a culturally competent approach to HIV awareness-raising in BME MSM is required. These findings shed light on the contexts in which HIV prevention efforts should be targeted to reach specific ethnic groups, as well as some of the potential syndemics that can increase HIV risk or undermine HIV outcomes in BME MSM patients.
\end{abstract}

\section{Introduction}

Both men who have sex with men (MSM) and black and minority ethnic (BME) groups experience significant sexual health inequalities. BME MSM face a double jeopardy in relation to sexual health. Black MSM are more likely to be diagnosed with a bacterial sexually transmissible infection (STI) than other ethnic groups, ${ }^{1}$ and are sixfold more likely to live with undiagnosed HIV than other MSM. ${ }^{2}$ According to Public Health England, ${ }^{3}$ there has been a greater than $82 \%$ increase in new HIV diagnoses among MSM of 'other' and mixed heritage. There is a relationship between HIV risk awareness and risk behaviour. ${ }^{4}$ However, there are no data concerning the level of HIV knowledge in BME MSM in the UK. This study explored the relationships between HIV knowledge and reported sexual health and sexual behaviour in this population. Moreover, it examined differences between specific ethnic groups in the BME MSM population in levels of HIV knowledge and sexual behaviour.

HIV incidence in BME communities is high; in 2015, two thirds of all new HIV diagnoses among heterosexuals in the UK were in BME individuals. ${ }^{5}$ Furthermore, it has been shown that BME people are more likely to be diagnosed at an advanced stage of HIV infection, which can severely undermine disease prognosis. ${ }^{2}$ In a case note review of 203 
BME MSM attending a London genitourinary medicine (GUM) clinic, ${ }^{6}$ it was found that BME MSM were more likely to report unprotected anal sex with casual male partners in the past 3 months, indicating higher risk of HIV acquisition in this group. Moreover, BME MSM were more likely to report high-risk sexual behaviour than other MSM, and higher rates of bacterial STIs were observed in Black African and Black Caribbean MSM than in other MSM.

BME MSM are more likely to have a history of substance abuse and less likely to have heard of biomedical HIV prevention approaches, namely post-exposure prophylaxis (PEP) and pre-exposure prophylaxis (PrEP), than other MSM. ${ }^{2}$ Furthermore, the same study reported that BME MSM were threefold more likely to test positive for HIV than other MSM. $^{2}$ It is important to ascertain whether specific BME groups are at higher risk than others. In an analysis of the GUM clinical activity dataset in England, ${ }^{7}$ it was found that, among all MSM attending GUM services, BME MSM were more likely to be diagnosed with an STI than other MSM. Furthermore, MSM of White mixed and Black African backgrounds were more likely to be diagnosed with HIV than other MSM.

A cross-sectional study of HIV, sexual risk and ethnicity in English MSM demonstrated higher HIV prevalence in Black MSM than in White MSM, and that South Asians are now just as likely as White MSM to report having HIV. ${ }^{8}$ In a study of uptake of and retention in HIV care among MSM by ethnic group, the UK Collaborative HIV Cohort Study Group ${ }^{9}$ found that BME MSM were more likely to be lost to follow-up after HIV diagnosis than White MSM (3.4\% vs 2.2\%, respectively), and that MSM of other or mixed ethnicity were most likely to be permanently lost to follow-up. Moreover, BME MSM were $18 \%$ less likely to initiate antiretroviral therapy (ART) than White MSM with a similar CD4 cell count. These studies highlight not only a higher prevalence of HIV in BME MSM, but also a higher risk of infection and onward transmission in this population.

Drug abuse is associated with HIV acquisition and onward transmission, ${ }^{10}$ partly due to its association with engagement in sexual risk-taking behaviours. ${ }^{11} \mathrm{~A}$ greater prevalence of drug use has been observed in HIV-positive than HIV-negative MSM. ${ }^{12}$ Moreover, epidemiological data manifest a higher prevalence of both drug use and HIV in ethnic minorities in the US. ${ }^{12}$ African American and Latino MSM may use drugs as a maladaptive strategy for coping with HIV infection and other psychological adversity. ${ }^{10}$ It is thus possible that there is a higher prevalence of drug use in HIV-positive MSM of BME background than in HIV-negative BME MSM. Research into BME MSM in the UK has been scarce, and none has explored the relationship between drug use and sexual risk in this population. ${ }^{11}$ The present study addresses this lacuna.

No previous study has compared levels of HIV knowledge, HIV risk behaviours and drug use in specific BME ethnic groups. First, it can be hypothesised that HIV knowledge will be lower in South Asian MSM than in those of other BME backgrounds. Given the historically low incidence of HIV in South Asian communities in the UK, this community has not traditionally been targeted in prevention campaigns. Moreover, there is an observable cultural taboo around open discussions of sex and sexuality, given the importance of family and cultural honour, precluding open discussions about sexual health and HIV. ${ }^{13}$ There is also an observed reluctance to engage with sexual health services among South Asian MSM, which may mean that they are less likely than Latinos and other ethnic groups to engage with sexual health services whose cultural norms are less prohibitive of sexuality-related issues. ${ }^{14}$ Second, it is possible that levels of drug use (as a strategy for coping with psychological and emotional adversity) differ between ethnic groups and that migrant MSM may adopt drug use more frequently than other groups. ${ }^{15}$ Feeling connected to a community, as many South Asian MSM do, ${ }^{13}$ can buffer the negative psychological effects of adversity. Conversely, as migrants, some Latin American MSM may perceive a lack of social support, which, in turn, 
may lead to the adoption of maladaptive strategies, like drug use. ${ }^{16}$

Prejudice and social exclusion, such as experiences of racism and homophobia, have been linked to engagement in sexual risk-taking behaviours. In a sample of Latino gay men, social exclusion was associated with participation in sexually 'difficult' situations. ${ }^{17}$ In another study of Black and Latino MSM, racism and homophobia were associated with unprotected anal intercourse with a serodiscordant or sero-unknown partner. ${ }^{18}$ In a survey of Latino MSM in the US, respondents who reported homophobia were more likely to report condomless receptive anal intercourse than those with no experience of homophobia. ${ }^{19}$ Homophobia from within one's ethnic ingroup can decrease a sense of connection with one's ethnic ingroup, which, in turn, can increase sexual risk-taking behaviours. ${ }^{20}$ Crucially, BME MSM may face homophobia from both their ethnic ingroup and the general population. ${ }^{21}$ South Asian MSM in particular experience an elevated cultural taboo in relation to their sexual orientation, which may affect patterns of sexual identity disclosure (or 'coming out'), as well as the contexts in which they seek sexual partners to avoid involuntary disclosure. ${ }^{13}$ More generally, perceived exclusion from multiple social groups can lead to feelings of marginalisation, leaving individuals with few sources of social support and sexual health information. This relationship has yet to be studied in BME MSM in the UK. However, it can be hypothesised that those ethnic groups with decreased social capital (such as Black people) may face perceive greater discrimination and exclusion than those with higher social capital, namely South Asians.

Despite the sexual health inequalities faced by BME MSM and high HIV incidence in this population, there has been limited empirical research into the possible HIV risk factors in this population. ${ }^{22}$ Accordingly, the present study explores the relationships between HIV knowledge and reported sexual health and sexual behaviour in a sample of BME MSM in the UK.

\section{Hypotheses}

On the basis of this review of existing research into BME MSM, the following hypotheses are proposed: (1) South Asians will possess less HIV knowledge than Black and Latino participants; (2) in terms of risk behaviours, Latinos will use drugs more frequently than South Asian and Black participants, whereas South Asians will use gay saunas more frequently than other ethnic groups; (3) HIV-positive BME MSM are more likely than HIVnegative BME MSM to use drugs; (4) Latino participants will be more engaged around sexual health issues (manifested by more frequent sexual health screening) than South Asian and Black participants; and (5) experiences of homophobia and racism will be more frequent among Black individuals and associated, across the board, with engagement in sexual risktaking behaviours.

\section{Ethics}

\section{METHODS}

This study received ethics approval from the Faculty of Health and Life Sciences Ethics Committee, De Montfort University, Leicester (Reference 1788).

\section{Participants}

Using a convenience sampling strategy, 538 BME MSM were recruited over an 18-month period through the following sexual health charities: NAZ Project London (London), Trade Sexual Health (Leicester) and Yorkshire MESMAC (Leeds). The sample consisted of service users and the networks of BME health and well-being coordinators at these organisations. Respondents were asked to indicate whether they have sex with men, women or both. Only those who indicated that they had sex with men were included in the study. 
Of the study cohort, $346(64 \%)$ self-identified as South Asian, 88 (16\%) selfidentified as Latin American, 76 (14\%) self-identified as Black, 13 (2\%) self-identified as mixed, and $15(3 \%)$ self-identified as other. In terms of age, $112(22 \%)$ respondents were aged between 18 and 25 years, $266(52 \%)$ were aged between 26 and 35 years, $113(22 \%)$ were aged between 36 and 50 years and $12(2 \%)$ were aged $\geq 50$ years.

In all, $304(60 \%)$ respondents believed themselves to be HIV negative, and $106(21 \%)$ had been diagnosed with HIV. Of all HIV-positive participants, 31 were South Asian (11\% of South Asian respondents), 14 were Black (28\% of Black respondents) and 61 were Latin American (74\% of Latin American respondents).

Sixty-three (12\%) respondents reported an STI diagnosis in the past month, including gonorrhoea $(n=19)$, chlamydia $(n=14)$ and syphilis $(n=6)$.

\section{Measures}

HIV knowledge was measured using an adapted version of the 18-item HIV knowledge questionnaire. ${ }^{23}$ This measure includes statements such as 'Not wearing a condom has no effect on my risk for HIV transmission'. Possible responses include 'True', 'False' and 'Unsure'. Each participant received a score based on the number of correct responses to each statement. The frequency of sexual health screening was measured using the following item: 'Within the last 12 months, how many times have you participated in a full sexual health screening?' and perceived sexual risk was measured using the following item: 'I would consider the type of sex I've had in the last 12 months to be [all safe/mostly safe etc.]'.

The frequency of sauna attendance was measured using the following item: 'How often do you go to saunas?' and the frequency of sex seeking on mobile applications was measured using the following item: 'In the last 12 months, how many times have you used mobile or online technology to arrange sex?' The frequency of drug use was measured using the following item: 'How often do you use drugs?' It is noteworthy that this item measures drug use in general, rather than drug use in sexualised settings (or 'chemsex'). The frequency of homophobia was measured using the following item: 'How often do you experience homophobia?' and the frequency of racism was measured using the following item: 'How often do you experience racism?' All variables were measured on a five-point Likert scale, except for the perceived sexual risk scale, which was measured on a four-point scale. Higher scores indicate higher levels of each variable (e.g. a high score on 'frequency of sauna attendance' $=$ high frequency of sauna attendance).

Unless indicated otherwise, data are presented as the mean \pm s.d.

\section{Tests of normality}

\section{RESULTS}

Kolmogorov-Smirnov tests were used to test the distribution of the variables in this study. Results showed that the following variables were not normally distributed: HIV knowledge $(D(538)=2.79, P<0.001)$, frequency of sex seeking on applications $(D(538)=5.14, P$ $<0.001)$, frequency of sauna use $(D(538)=6.15, P<0.001)$, attendance at sex parties $(D(538)=9.58, P<0.001)$, frequency of drug use $(D(538)=10.14, P<0.001)$, perceived risk $(D(538)=5.77, P<0.001)$, sexual health screening $(D(538)=6.01, P<0.001)$ and HIV testing in the past year $(D(538)=6.09, P<0.001)$. Because the variables of interest were not normally distributed, non-parametric analyses were used, including the Kruskal-Wallis and Spearman Rho tests.

\section{HIV knowledge}

Overall, participants scored a mean of $3.66 \pm 2.12$ (out of 10 ), indicating a very low level of HIV knowledge. There were no significant differences between age groups on levels of HIV 
knowledge. A Kruskal-Wallis test showed an effect of ethnicity on HIV knowledge $(H(4)=$ 70.97, $P<0.001)$. As indicated in Fig. 1, levels of HIV knowledge were lower in South Asians than in Black and Latino respondents $(3.2 \pm 1.9$ vs $4.47 \pm 2.25$ and $4.89 \pm 2.06$ respectively). The Kruskal-Wallis test also showed an effect of education on HIV knowledge $(H(2)=15.05, P=0.001)$, with a mean score of $4.12 \pm 2.19$ for participants who had completed university, compared with $3.40 \pm 1.88$ and $2.88 \pm 2.03$ for those who had not and had completed high school respectively. A Chi-squared test showed that South Asians were more likely to have completed high school and to have a university degree than Black and Latin American respondents $\left(\chi^{2}(8535)=23.77, P=0.003\right)$.

Scores were generally high on the perceived risk variable $(2.62 \pm 0.72,2.67 \pm 0.85$ and $2.8 \pm 1.1$ for South Asian, Latin American and Black participants respectively). Median splits were performed on the variable of perceived risk to compare high and low perceived risk groups. Of 538 participants, 213 fell into the low perceived risk group (40\%) and 42 fell into the high perceived risk group (8\%). A Kruskal-Wallis test demonstrated a significant effect of perceived risk on HIV knowledge $(H(1)=8.21, P<0.001)$, indicating that those who perceived their behaviour as least risky achieved significantly lower HIV knowledge scores $(3.81 \pm 1.97)$ than those who appraised their sexual behaviour as mostly unsafe or all unsafe ( $4.86 \pm 2.43$; Fig. 2). This suggests that there may be elements of inaccurate risk appraisal at play due to lack of knowledge.

\section{Sexual risk taking and sexual health behaviours}

A Kruskal-Wallis test examined the role of ethnicity on sexual risk-taking behaviours. In particular, we tested the difference between ethnic groups on four sexual risk-taking behaviours, namely frequency of sauna visits, frequency of sex-seeking on mobile applications, frequency of drug use and attendance at sex parties. The results revealed a significant multivariate main effect for ethnic group on attendance of sex parties $(H(4)=$ $12.02, P=0.017)$, frequency of sauna use $(H(4)=22.27, P<0.001)$, frequency of use of sex applications $(H(4)=12.69, P=0.013)$ and frequency of drug use $(H(4)=42.47, P<0.001)$.

As indicated in Fig. 3, significant univariate main effects for ethnicity were obtained for frequency of sauna attendance. Black participants frequented saunas significantly less often than Latino $(P=0.001)$ and South Asian $(P<0.001)$ participants. There were no significant differences between South Asian and Latino participants. Significant univariate main effects for ethnicity were obtained for frequency of sex seeking on mobile applications. Black participants used mobile applications for sex seeking significantly less frequently than Latino $(P=0.001)$ and South Asian $(P=0.01)$ participants. There were no significant differences between South Asian and Latino participants. Significant univariate main effects for ethnicity were obtained for frequency of drug use. Latino participants used drugs significantly more frequently than Black $(P=0.001)$ and South Asian $(P<0.001)$ participants. There were no significant differences in frequency of drug use between South Asian and Black participants. Significant univariate main effects for ethnicity were obtained for attendance at sex parties. In particular, Black participants attended sex parties significantly more frequently than both Latino $(P=0.01)$ and South Asian $(P=0.04)$ participants. However there were no significant differences in attendance between South Asian and Latino participants.

Frequency of engaging in the four sexual risk-taking behaviours was compared in HIV-positive and HIV-negative respondents. Of the four risky behaviours, there was only a significant difference between HIV-positive and -negative participants in the frequency of drug use $(1.72 \pm 1.05$ vs $1.29 \pm 0.73$ respectively; $H(1)=32.07, P<0.001)$. A further Chisquared test showed that Latin American participants were much more likely to be HIV positive than South Asian and Black participants $\left(\chi^{2}(4434)=139.34, P<0.001\right)$. 
As indicated in Fig. 4, Kruskal-Wallis test revealed a significant effect of ethnicity on the frequency of sexual health screening, which constitutes a sexual health behaviour $(H(4)=$ 45.15, $P<0.001$ ), indicating that Latin American respondents are significantly more likely to seek sexual health screening than other ethnic groups. A further test showed a significant effect of ethnicity on having had an HIV test in the past year, which is also a sexual health behaviour $(H(4)=23.89, P<0.001)$. Latin Americans were more likely to have been tested than Black and South Asian participants $(2.00 \pm 0.80$ vs $1.75 \pm 0.82$ and $1.66 \pm 0.80$ respectively).

\section{Discrimination}

On average, participants reported a mean score of $2.52 \pm 1.28$ on the frequency of homophobic experience and a mean score of $2.41 \pm 1.13$ on the frequency of racism, indicating that participants generally believed they faced homophobia and racism either 'sometimes' or 'often'. We established that there were significant differences in reported experiences of racism between South Asian, Black and Latin American participants (2.35 \pm $1.11,3.15 \pm 1.12$ and $2.07 \pm 0.96$ respectively; $H(4)=37.03, P<0.001)$. There were also significant differences in reported experiences with homophobia between South Asian, Black and Latin American participants $(2.52 \pm 1.29,2.92 \pm 1.23$ and 2.26 \pm 0.1 .2 respectively; $H(4)$ $=11.62, P=0.020$ ).

Spearman Rho's correlations showed that among South Asians there were positive correlations between homophobia and sex seeking on mobile applications $(r=0.12, P=$ $0.046)$ and sauna frequency $(r=0.16, P=0.004)$, as well as between racism and sex seeking on mobile applications $(r=0.15, P=0.009)$ and sauna frequency $(r=0.14, P=0.011)$. Among Black participants, there were positive correlations between racism experiences and frequency of sex seeking on mobile applications $(r=0.30, P=0.017)$ and between homophobia and frequency of sauna attendance $(r=0.26, P=0.046)$. Among Latinos, there was a positive correlation between racism and frequency of sex seeking on mobile applications $(r=0.24, P=0.025)$ and very significant positive correlations between racism and sauna frequency $(r=0.43, P<0.001)$ and between homophobia and frequency of sauna attendance $(r=0.33, P=0.002)$.

\section{DISCUSSION}

The data presented herein provide greater clarity on the potential antecedents of continued HIV incidence in BME MSM, in contrast with the $32 \%$ fall in new HIV diagnoses in other MSM in London that was reported at the end of 2016. ${ }^{24}$ A key starting-point for effective HIV prevention is promoting knowledge of HIV, principal transmission routes and prevention methods. Yet, in our sample of BME MSM, HIV knowledge appears to be low across the board. South Asian MSM in particular appear to possess the least knowledge of HIV. This can be attributed, in part, to the cultural taboo appended to overt discussions of sexuality and HIV, as well as the rejection of homosexuality in some cultural groups. ${ }^{14} \mathrm{We}$ did find a significant effect of education level on HIV knowledge, suggesting that educational settings can provide an important context for increasing awareness and understanding of HIV risk factors. Furthermore, it is concerning that those respondents who perceived their HIV risk to be low had the least HIV knowledge, because this casts doubt on their ability to formulate an accurate HIV risk appraisal. That is, they may be taking risks without realising. Individuals who do not perceive themselves to be at risk of HIV are less likely to test for $\mathrm{HIV}^{25}$ and more likely to live with undiagnosed HIV. ${ }^{2}$ This may explain, at least in part, the continued HIV incidence in this population. These data indicate an urgent need to focus HIV awareness-raising campaigns on BME MSM who face a significant HIV risk but who manifest decreased awareness and understanding of HIV. This will be challenging due to 
cultural taboos around sexuality and HIV and will necessitate a culturally competent approach to awareness raising. ${ }^{16}$

There was a high prevalence of both racism and homophobia in our sample of BME MSM, with Black respondents reporting the highest frequency of these forms of prejudice. Prejudice is aversive for both psychological well-being ${ }^{26}$ and, as demonstrated in studies conducted in the US, ${ }^{18}$ for sexual health outcomes in BME MSM. Experiences of prejudice may also affect the ways in which BME MSM seek sexual encounters with other men; indeed, there was a positive relationship between frequency of racism and sex seeking on mobile applications across all ethnic groups. It has been found that some MSM may perceive greater confidence and self-efficacy in relation to sex seeking and interactions with other men than in offline settings, ${ }^{27}$ which may explain the preference for this forum among BME MSM who have experienced racism (including from other MSM). Furthermore, research has shown that gay mobile applications are perceived to constitute a 'safer' sex for avoiding involuntary disclosure of one's sexual orientation, which itself may be associated with homophobia. ${ }^{27}$

There were significant differences in engagement in sexual risk-taking behaviours by ethnicity and HIV status. Latino respondents are more likely to frequent gay saunas and to report drug use. Unlike the Black and South Asian participants, Latinos in London are largely migrants and drug use is known to be more prevalent in migrant than non-migrant MSM in some countries. ${ }^{15}$ Furthermore, it is important to note that the majority of Latino respondents were HIV positive and that drug use (often as a maladaptive strategy for coping with psychological adversity) is more prevalent in HIV-positive MSM than in HIV-negative MSM. ${ }^{12}$ Black respondents are more likely to attend gay parties. Latino and South Asian MSM are more likely to seek sex on mobile applications. Furthermore, HIV-positive BME MSM are more likely to report drug use than those who are HIV negative. These findings provide some insight into the contexts in which HIV prevention efforts should be targeted if they are to reach specific BME subgroups (Latino, South Asian or Black). HIV prevention interventions that target particular ethnic groups in the settings in which they habitually seek sexual encounters may be more effective than a blanket approach. In view of the evidence that South Asian and Black MSM are less likely to seek sexual health screening, there is a need to engage these groups around the importance of screening. Furthermore, given the well documented relationship between drug use and decreased engagement with HIV care, poor ART adherence and overall HIV outcomes, ${ }^{28}$ HIV care for BME MSM, and especially for Latino MSM, should incorporate the possibility of potential drug use. ${ }^{16}$

\section{Limitations}

This survey has several limitations that ought to be addressed in subsequent research. First, although this study has a large sample size of 538 BME MSM recruited from three distinct cities, most respondents (68\%) were South Asian MSM. It is hoped that future research will include larger subsamples of other ethnic groups, including Latino respondents, in order to provide a fuller picture of HIV knowledge, sexual health and sexual behaviour in BME MSM. Second, the survey did not measure actual engagement in sexual risk-taking behaviours, which makes it difficult to conclude that respondents who perceive their behaviour as low risk are actually engaging in risky behaviours. The lack of these behavioural data also impedes the corroboration of previous research that has found a relationship between prejudice and sexual risk taking. Thus, actual engagement in HIV risk behaviours would be an insightful variable to consider in future work. Third, it is acknowledged that the relationship between risk awareness and engagement in risky behaviour is complex and likely to be mediated by factors that were not measured in this study. These factors include self-esteem, self-efficacy, partner status and other identity concerns ${ }^{13}$ and they should be incorporated in future research into sexual risk among BME 
MSM.

\section{Conclusion}

HIV knowledge is unacceptably low in BME MSM. This may undermine individuals' ability to formulate an accurate HIV risk appraisal and lead to continued HIV incidence in this population. Furthermore, the results suggest that engagement with sexual health services (itself beneficial for sexual health awareness and screening) is higher for some ethnic groups than for others, highlighting the need to increase engagement in those groups. A culturally competent approach to HIV awareness raising in BME MSM is required. This study demonstrates significant differences in HIV knowledge, sexual health and sexual behaviours between the three ethnic groups surveyed. These findings shed light on the contexts in which HIV prevention efforts should be targeted, as well as some of the potential syndemics that can increase HIV risk or undermine HIV outcomes in BME MSM patients. Given the distinct patterns of perceived HIV risk and engagement in HIV risk behaviours in distinct BME ethnic groups, we argue that a 'one size fits all' approach to HIV prevention is unlikely to be effective. Rather, it is important that cultural differences are acknowledged and that a culturally nuanced HIV prevention approach is deployed.

\section{REFERENCES}

1. Dougan S, Elford J, Rice B et al. Epidemiology of HIV among black and minority ethnic men who have sex with men in England and Wales. Sex Transm Infect. 2005;81(4):345-350.

2. Millett GA, Peterson JL, Flores SA et al. Comparisons of disparities and risks of HIV infection in black and other men who have sex with men in Canada, UK, and USA: A meta-analysis. Lancet. 2012;380(9839):341-348. doi:10.1016/S0140-6736(12)60899$\mathrm{X}$

3. Public Health England. National HIV surveillance data tables. Available from: https://www.gov.uk/government/statistics/hiv-annual-data-tables [Accessed on 20th December 2017]

4. Leidner AJ, Shaw WD, Yen, ST. An historical perspective on health-risk awareness and unhealthy behaviour: Cigarette smoking in the United States 1949-1981. Health Expect. 2016;18(6):2720-30.

5. Public Health England. Sexually Transmitted Infections in England, 2015. Public Health England. Available from:

https://www.gov.uk/government/uploads/system/uploads/attachment_data/file/617030 /England_STI_Slide_Set_2016.pdf [Accessed on 20 ${ }^{\text {th }}$ December 2017]

6. Soni S, Bond, K, Fox, E et al. Black and minority ethnic men who have sex with men: A London genitourinary medicine clinic experience. Int J STD AIDS. 2008;19(9):617-619. doi:10.1258/ijsa.2008.008039 
7. Mohammed H, Furegato M, Hughes G. Inequalities in sexually transmitted infection risk among black and minority ethnic men who have sex with men in England. Sex Transm Infect. 2016; 92(Suppl 1): A1-A106. Available from:

http://sti.bmj.com/content/92/Suppl_1/A42.1

8. Hickson F, Melendez-Torres GJ, Reid D. H et al. HIV, sexual risk and ethnicity among gay and bisexual men in England: survey evidence for persisting health inequalities. Sex Transm Infect. 2017. doi: 10.1136/sextrans- 2016-052800

9. United Kingdom Collaborative HIV Cohort Study Group. Uptake and outcome of combination antiretroviral therapy in men who have sex with men according to ethnic group: The UK CHIC Study. J Acquir Immune Defic Synd. 2012; 59(5):523-9.

10. Pence BW, Thielman NM, Whetten K et al. Coping strategies and patterns of alcohol and drug use among HIV-infected patients in the United States Southeast. AIDS Patient Care STDS. 2008; 22(11), 869-87. doi:10.1089/apc.2008.0022

11. Melendez-Torres GJ, Bourne A. Illicit drug use and its association with sexual risk behaviour among MSM: More questions than answers?. Curr Opin Infect Dis. 2016; 29(1):58-63. doi:10.1097/QCO.0000000000000234.

12. Daskalopoulou M, Rodger A, Phillips AN, et al. Recreational drug use, polydrug use, and sexual behaviour in HIV-diagnosed men who have sex with men in the UK: results from the cross-sectional ASTRA study. Lancet HIV. 2014;1(1):e22-31.

13. Jaspal R. Enhancing sexual health, self-identity and wellbeing among men who have sex with men: A guide for practitioners.2018. London: Jessica Kingsley Publishers.

14. Weston HJ. Public honour, private shame and HIV: issues affecting sexual health service delivery in London's South Asian communities. Health \& Place. 2003;9(2):109-117

15. Yang X, Derlega VJ, Luo H. Migration, behaviour change and HIV/STD risks in China. AIDS Care. 2007;19:282-288.

16. Jaspal R, Williamson I. Identity management strategies among HIV-positive Colombian gay men in London. Culture, Health and Sexuality: An International Journal for Research, Intervention and Care. 2017;19(2):1374-1388. doi:10.1080/13691058.2017.1314012

17. Diaz RM, Ayala G, Bein E. Sexual risk as an outcome of social oppression: Data from a probability sample of Latino gay men in three U.S. cities. Cult Divers Ment Health. 2004;10(3):255-267. doi:10.1037/1099-9809.10.3.255. 
18. Ayala G, Bingham T, Kim, J et al. Modeling the impact of social discrimination and financial hardship on the sexual risk of HIV among Latino and Black men who have sex with men. Am J Public Health. 2012;102(S2):242-249. doi:10.2105/AJPH.2011.300641 .

19. Mizuno Y, Borkowf C, Millett GA et al. Homophobia and racism experienced by Latino men who have sex with men in the United States: Correlates of exposure and associations with HIV risk behaviors. AIDS Behav. 2012;16(3):724-735. doi:10.1007/ s10461-011-9967-1.

20. O’Donnell L, Agronick G, Doval AS, Duran R, Myint-U A, Stueve A. Ethnic and gay community attachments and sexual risk behaviors among urban Latino young men who have sex with men. AIDS Educ Prev. 2002;14(6):457-71.

21. Jaspal R, Siraj A. Perceptions of 'coming out' among British Muslim gay men. Psychology and Sexuality. 2011;2(3):183-197. doi:10.1080/19419899.2010.526627.

22. Fish J, Papaloukas P, Jaspal R et al. Equality in sexual health promotion: a systematic review of effective interventions for black and minority ethnic men who have sex with men. BMC Public Health. 2016;16:810. doi:10.1186/s12889-016-3418-x.

23. Carey MP, Schroder KE. Development and psychometric evaluation of the brief HIV Knowledge Questionnaire. AIDS Educ Prev. 2002;14(2):172-182. doi:10.1521/aeap.14.2.172.23902 .

24. Brown AE, Mohammed H, Ogaz D et al. Fall in new HIV diagnoses among men who have sex with men (MSM) at selected London sexual health clinics since early 2015: Testing or treatment or pre-exposure prophylaxis (PrEP)?.Euro Surveill. 2017;22(25): 30553.

25. Stephenson R, White D, Darbes L et al. HIV testing behaviors and perceptions of risk of HIV infection among MSM with main partners. AIDS Behav. 2015;19(3):553-60. doi: 10.1007/s10461-014-0862-4.

26. Meyer IH. Prejudice, social stress, and mental health in lesbian, gay, and bisexual populations: Conceptual issues and research evidence. Psychol Bull. 2003;129:674697.

27. Jaspal R. Gay men's construction and management of identity on Grindr. Cult Health Sex. 2017;21(1):187-204. doi.org/10.1007/s12119-016-9389-3.

28. Hinkin CH, Hardy DJ, Mason KI et al. Medication adherence in HIV-infected adults: effect of patient age, cognitive status, and substance abuse. AIDS. 2004;18(Suppl 1):S19-S25. 


\section{Figures}

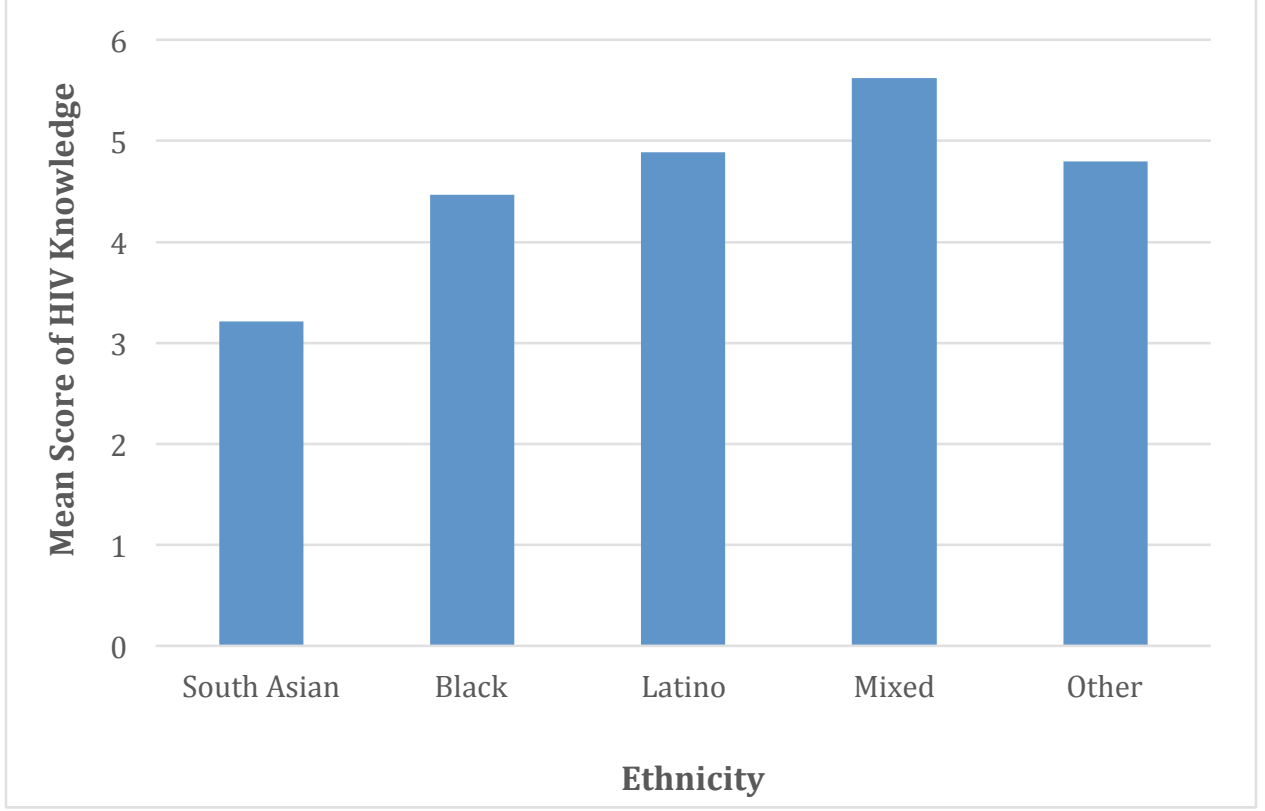

Figure 1. Mean Scores of HIV knowledge by ethnic group

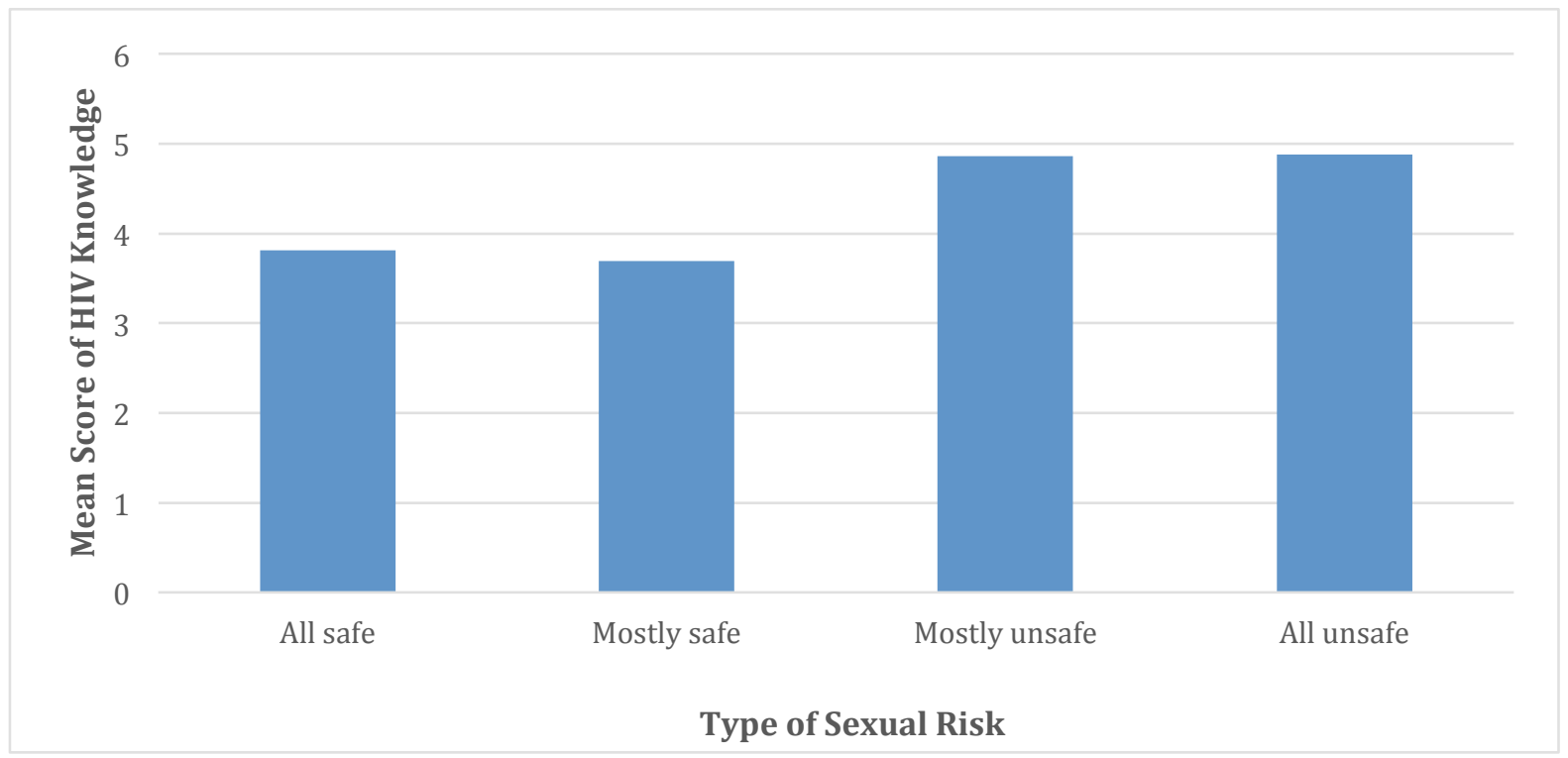

Figure 2. Mean Scores of HIV Knowledge by sexual risk type 


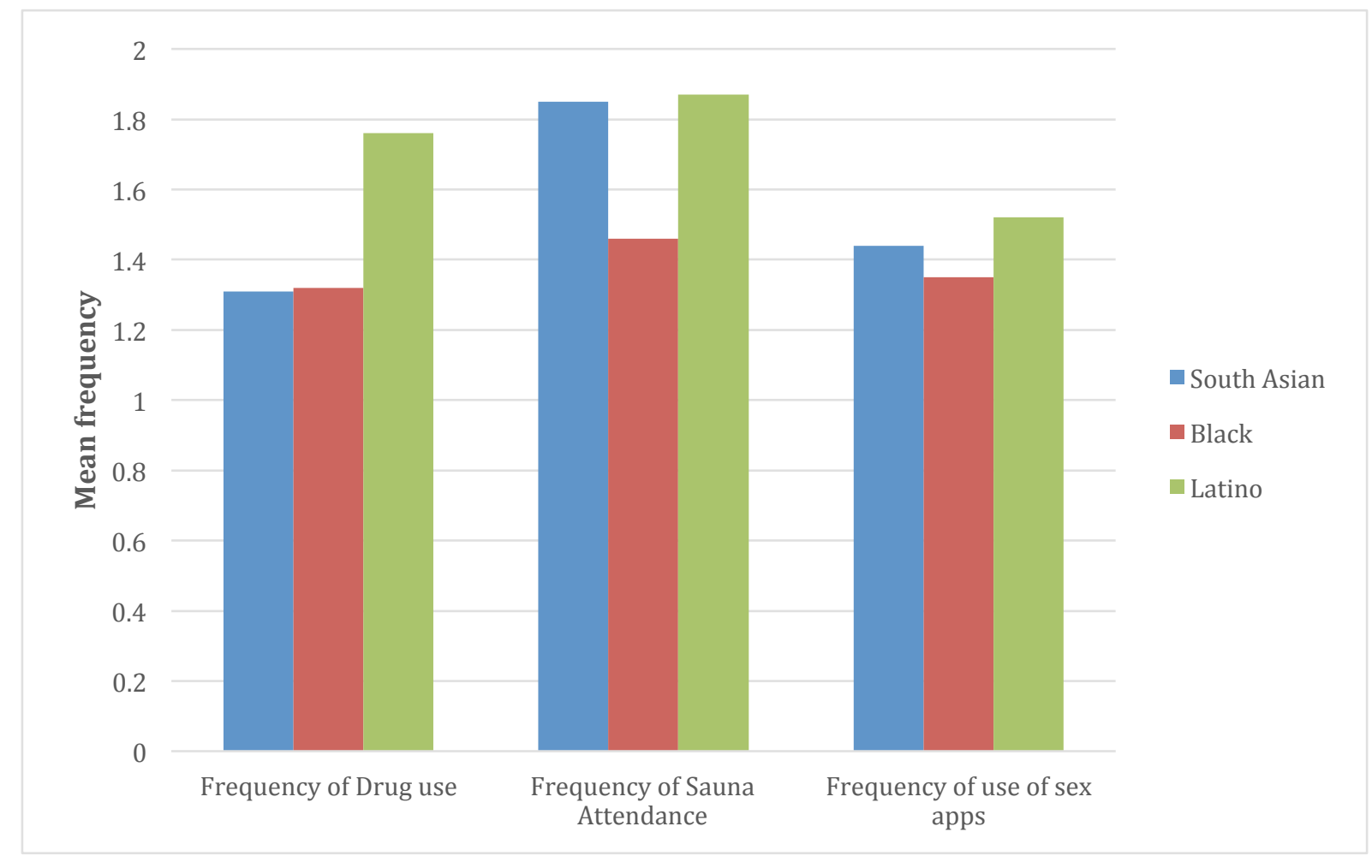

Figure 3. Mean frequencies of drug use, sauna attendance and use of sex-seeking applications by ethnic group 


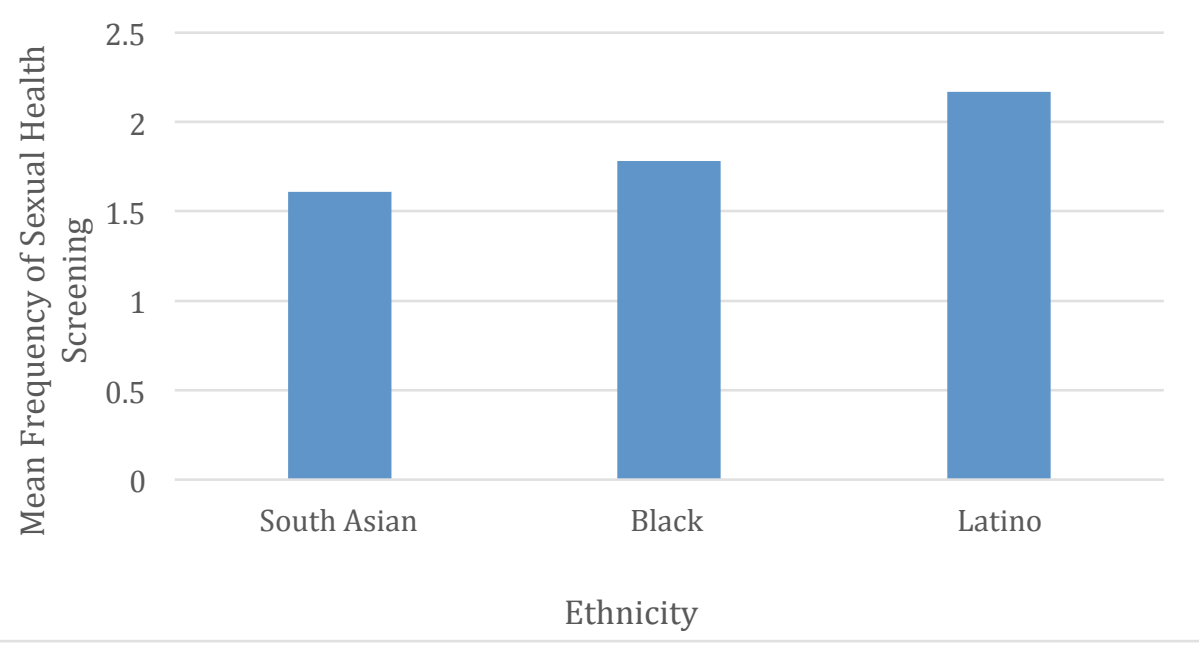

Figure 4. Mean frequency of sexual health screening in the last year by ethnic group 